\title{
DYNAMICAL MODEL FOR SPINDOWN OF SOLAR-TYPE STARS
}

\author{
Aditi SOOD ${ }^{1}$, EUN-Jin Kim ${ }^{1}$, AND Rainer Hollerbach ${ }^{2}$ \\ ${ }^{1}$ School of Mathematics and Statistics, University of Sheffield, Sheffield S3 7RH, UK \\ ${ }^{2}$ Department of Applied Mathematics, University of Leeds, Leeds LS2 9JT, UK \\ Received 2015 October 11; revised 2016 August 26; accepted 2016 September 10; published 2016 November 21
}

\begin{abstract}
After their formation, stars slow down their rotation rates by the removal of angular momentum from their surfaces, e.g., via stellar winds. Explaining how this rotation of solar-type stars evolves in time is currently an interesting but difficult problem in astrophysics. Despite the complexity of the processes involved, a traditional model, where the removal of angular momentum by magnetic fields is prescribed, has provided a useful framework to understand observational relations between stellar rotation, age, and magnetic field strength. Here, for the first time, a spindown model is proposed where loss of angular momentum by magnetic fields evolves dynamically, instead of being prescibed kinematically. To this end, we evolve the stellar rotation and magnetic field simultaneously over stellar evolution time by extending our previous work on a dynamo model which incorporates nonlinear feedback mechanisms on rotation and magnetic fields. We show that our extended model reproduces key observations and is capable of explaining the presence of the two branches of (fast and slow rotating) stars which have different relations between rotation rate $\Omega$ versus time (age), magnetic field strength $|B|$ versus rotation rate, and frequency of magnetic field $\omega_{\text {cyc }}$ versus rotation rate. For fast rotating stars we find that: (i) there is an exponential spindown $\Omega \propto e^{-1.35 t}$, with $t$ measured in Gyr; (ii) magnetic activity saturates for higher rotation rate; (iii) $\omega_{\text {cyc }} \propto \Omega^{0.83}$. For slow rotating stars we find: (i) a power-law spindown $\Omega \propto t^{-0.52}$; (ii) that magnetic activity scales roughly linearly with rotation rate; (iii) $\omega_{\text {cyc }} \propto \Omega^{1.16}$. The results obtained from our investigations are in good agreement with observations. The Vaughan-Preston gap is consistently explained in our model by the shortest spindown timescale in this transition from fast to slow rotators. Our results highlight the importance of self-regulation of magnetic fields and rotation by direct and indirect interactions involving nonlinear feedback in stellar evolution.
\end{abstract}

Key words: evolution - stars: activity - stars: magnetic field - stars: rotation - stars: solar-type

\section{INTRODUCTION}

Spindown of stars is one of the most debated and interesting issues in astrophysics. Stellar rotation rate is the key parameter which is believed to affect the spindown process. Spindown is not only influenced by stellar properties such as mass, radius, and age, but also depends upon the evolution of stellar magnetic fields and their interaction with the stellar atmosphere (Scholz 2008). After their formation from interstellar clouds, which involves various internal changes, stars undergo rotational evolution in different stages (Keppens et al. 1995; Tassoul 2000), briefly summarized as follows. During early pre-main sequence evolution, the contraction that occurs in the star along with other various internal structural changes lead it to spin-up. Also, owing to diverse internal changes, a radiative core develops which rotates faster than the convective envelope. Coupling between the radiative core and convective envelope should be strong enough for angular momentum to be constantly transferred from the core to the envelope. This persistent supply of angular momentum reduces the amount of differential rotation produced in the star. By the time the star reaches late pre-main sequence or early main sequence, rotational evolution is modified by the stellar wind. Angular momentum loss via the stellar wind gradually decelerates and stops the spin-up of the convective envelope toward the end of late pre-main sequence phase, and causes a fast spindown of the envelope on the main sequence. The timescale at which the decoupling of the core and envelope occurs is observed to be very rapid (Keppens et al. 1995). With increasing rotation, the timescale for angular momentum loss through the stellar wind decreases and affects the magnetic field strength.
Consequently, for rapidly rotating stars, the magnetic field strength does not increase beyond a critical value at a certain rotation rate and instead becomes independent of rotation no matter how rapid. When the convection zone spins down toward the end of the pre-main sequence, the magnetic field strength is believed to scale linearly with rotation rate in the case of slow rotating stars.

Based upon the whole spindown process, stars are often classified into two groups: fast and slow rotating (Saar \& Brandenburg 1999, Brandenburg et al. 1998, Barnes 2003; Pizzolato et al. 2003; Mamajek \& Hillenbrand 2008; Wright et al. 2011; Vidotto et al. 2014). The existence of two branches of stars exhibiting different dependences of cyclic variation of stellar magnetic activity, expressed as cycle period $P_{\mathrm{cyc}}$ on rotation period $P_{\text {rot }}$, was confirmed by Brandenburg et al. (1998), and later by Saar \& Brandenburg (1999). We note that a relationship between cycle period and rotation period was first established by Noyes et al. (1984) as $P_{\text {cyc }} \propto P_{\text {rot }}^{n}$ with $n=1.25 \pm 0.5$. Brandenburg et al. showed all young, active, and fast rotating stars lie on one branch, namely the active branch $(A)$ with scaling exponent $n=0.80$, while all old, inactive, and slow rotating stars lie on another branch, namely the inactive branch (I) with scaling exponent $n=1.15$ (Charbonneau \& Saar 2001; Saar \& Brandenburg 2001). Furthermore, stars on the $A$ branch experience rapid spindown for which the rotation rate $\Omega$ is related to time/age with an exponential law given as $\Omega \propto e^{m t}$, where $m$ is a negative constant, and in this case magnetic activity is found to be saturated, that is, it becomes independent of rotation rate for rapidly rotating stars. Stars on the $I$ branch undergo a very slow spindown with a power-law dependence as $\Omega \propto t^{-1 / 2}$, known 
as power-law spindown (Skumanich 1972), and in this case magnetic activity is thought to scale linearly with rotation rate. The relationship between magnetic activity and rotation rate is important for understanding the physical process responsible for spindown of a star and was first determined by Pallavicini et al. (1981), while Micela et al. (1985) observed that this relationship does not hold for rapidly rotating stars. We note that the regime where magnetic activity increases linearly with rotation rate is termed the "unsaturated (non-saturated) regime," while the regime where magnetic activity becomes independent of rotation rate is termed the "saturated regime" in observational studies (e.g., Pizzolato et al. 2003; Mamajek \& Hillenbrand 2008; Wright et al. 2011; Vidotto et al. 2014).

One of the challenges in explaining spindown is the existence of a gap between the two branches of stars. During spindown, the star suddenly jumps from the $A$ to the $I$ branch, creating a gap between the two branches where stars are sparse. This gap was first observed by Vaughan \& Preston (1980) and is now known as the $V-P$ gap. Various mechanisms have so far been proposed for this gap, but the underlying physics is still an open question. Some of the previous suggestions are as follows. Durney et al. (1981) advocated a change in magnetic field morphology from complex to simple at the time when rotation decreases to a certain value. Saar (2002) proposed that the existence of two distinct branches of stars could be due to changes in differential rotation, $\alpha$-effect, and meridional flow speed (which is proportional to $\Omega$ in case of flux transport models, e.g., see Dikpati \& Charbonneau 1999) with stellar rotation rate. Barnes (2003) studied period-color diagrams of open clusters and suggested that the transition from the convective (fast rotators) to the interface sequence (slow rotators) is due to the shear produced during the decoupling of the core and envelope. This shear gives rise to large-scale magnetic fields, and recoupling of the core and convection zone shifts the star from the convective sequence to the interface sequence. Structural changes in large-scale magnetic fields (Donati \& Cameron 1997), changes in dynamo action (BöhmVitense 2007), and manifestation of different dynamos for different stars (Wright et al. 2011) were also proposed as possible reasons for the $V-P$ gap.

Given the complexity of the spindown problem, which depends upon various parameters such as rotation rate, evolution of magnetic fields, and differential rotation, it is not possible to study a full magnetohydrodynamic model over the entire spindown timescale (e.g., from $10^{7}$ to $10^{9} \mathrm{yr}$ ). Therefore, various simplified models have been utilized to understand stellar evolution (e.g., Weber \& Davis 1967; Mestel 1968; Mestel \& Spruit 1987; Kawaler 1988; Cohen et al. 2009; Cranmer \& Saar 2011; Matt et al. 2012; Garraffo et al. 2015; Johnstone et al. 2015; Matt et al. 2015). One such model is the double zone model (DZM) which is based upon the stellar wind torque law (Weber \& Davis 1967; Mestel 1968; Belcher \& MacGregor 1976; Kawaler 1988). The main feature of this model is the bifurcated expression considered for the torque acting on the star (depending on the critical rotation rate) due to its magnetized stellar wind. MacGregor \& Brenner (1991) used this DZM model for coupled (ordinary differential) equations for the rotation rates of the stellar envelope and radiative core, where the angular momentum loss is prescribed according to the relation between rotation and magnetic field strength. To understand the distribution of stellar rotation at different ages, Keppens et al. (1995) extended this parameterized model to describe the evolution of a single star by taking into account angular momentum exchange, moment of inertia evolution, and torque exerted on the core and envelope, which cause changes in angular momentum. Since then, this model was extended by considering different initial conditions and tested against various observations in the spindown process (Krishnamurthi et al. 1997; Irwin \& Bouvier 2009; Denissenkov et al. 2010; Leprovost \& Kim 2010; Spada et al. 2011; Reiners \& Mohanty 2012; Gallet \& Bouvier 2013; Epstein \& Pinsonneault 2014). Apart from DZM there are other models such as the symmetrical empirical model (SEM, Barnes 2010; Barnes \& Kim 2010) and the metastable dynamo model (MDM, Brown 2014). Both SEM and MDM utilize observational data of two different sequences of stars to finetune their models and thus are descriptive rather than explanatory. Specifically, SEM uses the different period evolution of the two sequences (for active and inactive stars) depending on whether the rotation rate is above/below the critical value and fits the parameters from period-color diagrams by obtaining a best fit to the observational data. Unlike SEM, MDM uses one function for all rotation rates but two different coupling constants. By fine-tuning the values of these two coupling constants and the probability for the transition from small to large couplings, MDM improves the agreement with observations over SEM. Although it is still empirical, MDM is remarkable in introducing into a spindown model a threshold-like behavior with different coupling constants and their probabilistic nature. Possible mechanisms for these different coupling constants was later provided, e.g., by invoking a change in magnetic complexity (Garraffo et al. 2015; Réville et al. 2015). Recently, Matt et al. (2015) proposed a stellar wind torque model which reproduces the shape of the upper and lower envelopes, which corresponds to the transition region between the saturated and unsaturated regimes by explaining the mass-dependence of stellar magnetic and wind properties.

In this paper, we propose for the first time a dynamical model of spindown where the loss of angular momentum by magnetic field is treated dynamically, instead of being prescribed kinematically. To this end, we evolve the stellar rotation and magnetic field simultaneously over the stellar evolution time by extending our previous work (Sood \& Kim 2013 , 2014) which incorporates nonlinear feedback mechanisms on rotation and magnetic fields via $\alpha$-quenching and magnetic flux losses as well as mean and fluctuating rotation. We note that Sood \& $\operatorname{Kim}(2013,2014)$ demonstrated that nonlinear feedback plays a vital role in the generation and destruction of magnetic fields as well as self-regulation of the dynamo. In particular, it was found that a dynamic balance is required not only in the generation and destruction of magnetic fields but also in the fluctuating and mean differential rotation for the working of dynamo near marginal stability; their results were consistent with observations such as the linear increase in cycle frequency of the magnetic field with moderate rotation rates, levelling off of magnetic field strength with sufficiently large rotation rates, and quenching of shear. We extend this model to simultaneously evolve rotation and magnetic fields over the spindown timescale of a star, since their dynamics are closely linked through angular momentum loss and dynamo. That is, the angular momentum loss responsible for the spindown of a star depends upon magnetic fields which in turn are affected by rotation rates. We show that this model has 
the capability of explaining the existence of the two branches of stars, different rotation rate dependence of cycle frequency of the magnetic fields for these two branches, and the gap between the two branches, reproducing the main observations. By extending our previous work, our model is designed in such a way that it has the essential ingredients mentioned above to explain the complex process of spindown of solar-type stars, and to highlight the importance of nonlinear feedback in this process.

\section{THE MODEL}

We propose a dynamical model for the evolution of rotation rate and magnetic field in spindown by extending a previous nonlinear dynamo model (Weiss et al. 1984; Sood \& Kim 2013, 2014). In particular, Sood \& $\operatorname{Kim}(2013,2014)$ incorporated various nonlinear transport coefficients such as $\alpha$-quenching and flux losses and took the control parameter $D$, known as the dynamo number, to scale with rotation rate as $D \propto \Omega^{2}$. The model equations in dimensionless form are given as

$$
\begin{gathered}
\dot{A}=\frac{2 D B}{1+\kappa\left(|B|^{2}\right)}-\left[1+\lambda_{1}\left(|B|^{2}\right)\right] A, \\
\dot{B}=i\left(1+w_{0}\right) A-\frac{1}{2} i A^{*} w-\left[1+\lambda_{2}\left(|B|^{2}\right)\right] B, \\
\dot{w_{0}}=\frac{1}{2} i\left(A^{*} B-A B^{*}\right)-\nu_{0} w_{0} . \\
\dot{w}=-i A B-\nu w .
\end{gathered}
$$

Here, the poloidal magnetic field is represented by $A$, the toroidal magnetic field is given by $B, w_{0}$ is the mean differential rotation, and $w$ is the fluctuating differential rotation; $A, B$ and $w$ are complex variables whereas $w_{0}$ is real. We note that $w_{0}$ has zero frequency, and $w$ has twice the frequency of $A$ and $B$. The complex conjugates of $A$ and $B$ are denoted by $A^{*}$ and $B^{*}$. In this model, $A$ is generated by $B$ (e.g., $\alpha$-effect through helicity) which is assumed to be proportional to the rotation rate $\Omega$ (see Equation (1)). Equation (2) represents the generation of $B$ by $A$, where the quenching of the $\Omega$-effect is incorporated by the total shear $1+w_{0}$. The differential rotation is inhibited by the tension in the magnetic field lines via the Lorentz force and causes the quenching of the $\Omega$-effect. Due to back-reaction, the total shear is reduced from 1 to $1+w_{0}<1$ as $w_{0}$ is always negative and is given by $1+w_{0}=\Delta \Omega / \Omega$. The generation of $w_{0}$ and $w$ is represented by Equations (3) and (4), respectively. $\nu_{0}$ and $\nu$ represent the viscosity of $w_{0}$ and $w$, respectively; $\kappa, \lambda_{1}$ and $\lambda_{2}$ are constant parameters which represent the strength of nonlinear feedback due to the Lorentz force by the magnetic field and enhanced magnetic dissipation (e.g., magnetic flux loss). In particular, $\kappa$ represents the efficiency of the quenching of the $\alpha$-effect while $\lambda_{1}$ and $\lambda_{2}$ represent the efficiency in the poloidal and toroidal magnetic flux losses, respectively (see Sood \& Kim 2013 for full details).

To understand the evolution of rotation rate and magnetic field in the spindown of solar-type stars, we extend this model by upgrading $\Omega$ from a kinematically prescribed to a dynamic variable. To this end, we first replace $D$ by the square of the time-dependent rotation rate $\Omega(t)$ in Equation (1):

$$
\dot{A}=\frac{2 \Omega^{2} B}{1+\kappa\left(|B|^{2}\right)}-\left[1+\lambda_{1}\left(|B|^{2}\right)\right] A,
$$

where $\Omega$ is real. Second, we need to include an additional equation for the evolution of $\Omega(t)$ to model the spindown of a star by the loss of angular momentum due to magnetic fields. While the latter depends on many factors such as the mass flux and geometry and complexity of the magnetic fields (e.g., Garraffo et al. 2016), for example, the Alfvén radius over which it acts as a rotational brake and the latitude at which the mass release occurs, for simplicity, we incorporate their overall effects in our dynamical model by the ansatz that the decay rate of $\Omega$ is proportional to the strength of the magnetic fields as $\varepsilon_{1}|B|^{2}+\varepsilon_{2} \frac{|A|^{2}}{\Omega}$ with the two tunable parameters $\epsilon_{1}$ and $\epsilon_{2}$. Here, $|B|$ represents the strength of the toroidal magnetic field and $\frac{|A|}{\Omega}$ is the strength of the poloidal magnetic field in physical units due to our non-dimensionalization (see Sood \& Kim 2013). Our empirical model is thus described by the following equation for $\Omega$ :

$$
\dot{\Omega}=-\varepsilon_{1}|B|^{2} \Omega-\varepsilon_{2} \frac{|A|^{2}}{\Omega^{2}} \Omega .
$$

Equation (6) represents the overall spindown of the star as a whole due to the loss of angular momentum through magnetic fields. The constant parameters $\varepsilon_{1}$ and $\varepsilon_{2}$ represent the efficiency of angular momentum loss via toroidal and poloidal magnetic fields, respectively, which are taken to be independent in general, given the uncertainty regarding the precise role of these fields in spindown. Equation (6) is motivated to capture the key feature of the previous model (e.g., DZM) where the dependence of the angular momentum loss on $\Omega$ is roughly proportional to $\Omega^{3}$ for slowly rotating stars (below the critical rotation rate), and to $\Omega$ for fast rotating stars (above the critical rotation rate). Specifically, for fast rotating stars with the rotation rate above the critical value, $|B|$ and $|A|$ become independent of $\Omega$, and Equation (6) reduces to $\dot{\Omega} \propto-\Omega$, resulting in the exponential decay of $\Omega$ with time. On the other hand, for slow rotating stars, $\dot{\Omega} \sim-\Omega^{3}$ would be reproduced should the magnetic field increase linearly with $\Omega$ as $|B|,|A| \sim \Omega$ (see Section 3.2 for the scaling relation). To summarize, our extended model consists of Equations (2)-(4) and (5), (6), where Equations (2)-(4) are the same as in our previous model, Equation (5) is the modified form of Equation (1), and (6) is a new equation to model the time evolution of $\Omega$.

This system is investigated taking $\nu=0.5, \nu_{0}=35.0$, $\kappa=0.025, \lambda_{1,2}=1.125$, and $\varepsilon_{1,2}=3.5 \cdot 10^{-5}$. The parameters $\nu, \nu_{0}, \kappa$, and $\lambda_{1,2}$ are much the same as in our previous work (Sood \& Kim 2013, 2014). As can be seen from Equation (6), the two new parameters $\varepsilon_{1,2}$ control the rate of the spindown process. The value $3.5 \cdot 10^{-5}$ was chosen as it yields an overall spindown timescale of several Gyr; larger (smaller) values of $\varepsilon_{1,2}$ were also investigated, and yielded qualitatively the same dynamics, simply occurring on shorter (longer) timescales. In particular, we have checked that qualitatively similar results are obtained in the limiting cases where $\epsilon_{1}=0$ or $\epsilon_{2}=0$. Correspondingly, the dimensionless timescales are such that 

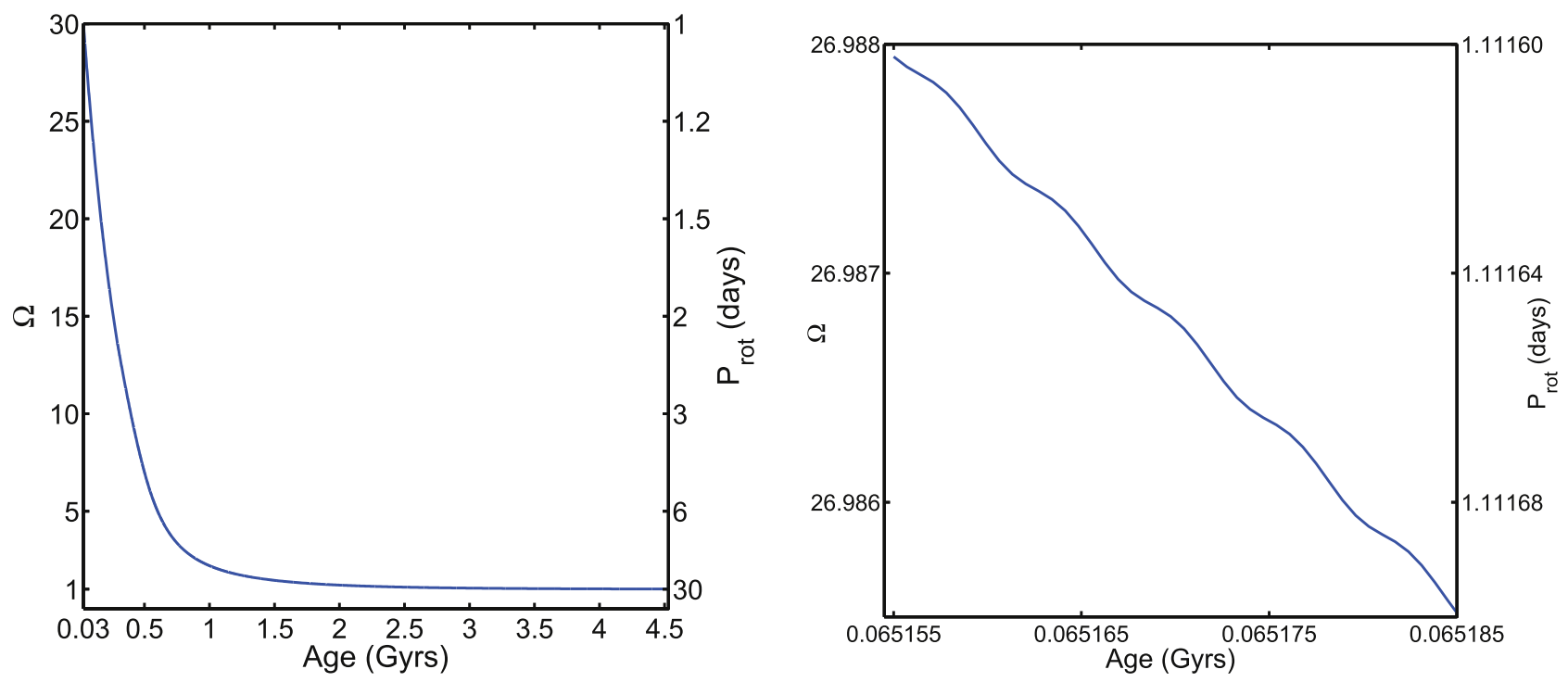

Figure 1. Left panel shows $\Omega$ as a function of age over the full range [0.03, 4.53] Gyr. As noted in Section 3, we scale our dimensionless time in physical units by the age of the present-day Sun of $4.5 \mathrm{Gyr}$, while our dimensionless rotation, represented on the vertical axis, is scaled with thirty times solar rotation to obtain the rotation period $\left(P_{\text {rot }}\right)$ in days, which is depicted on the right side of the $y$-axis. The right panel shows a zoomed-in view for age $=[0.065155,0.065185] \mathrm{Gyr}$, and reveals the presence of fluctuations superimposed on the general spindown trend.
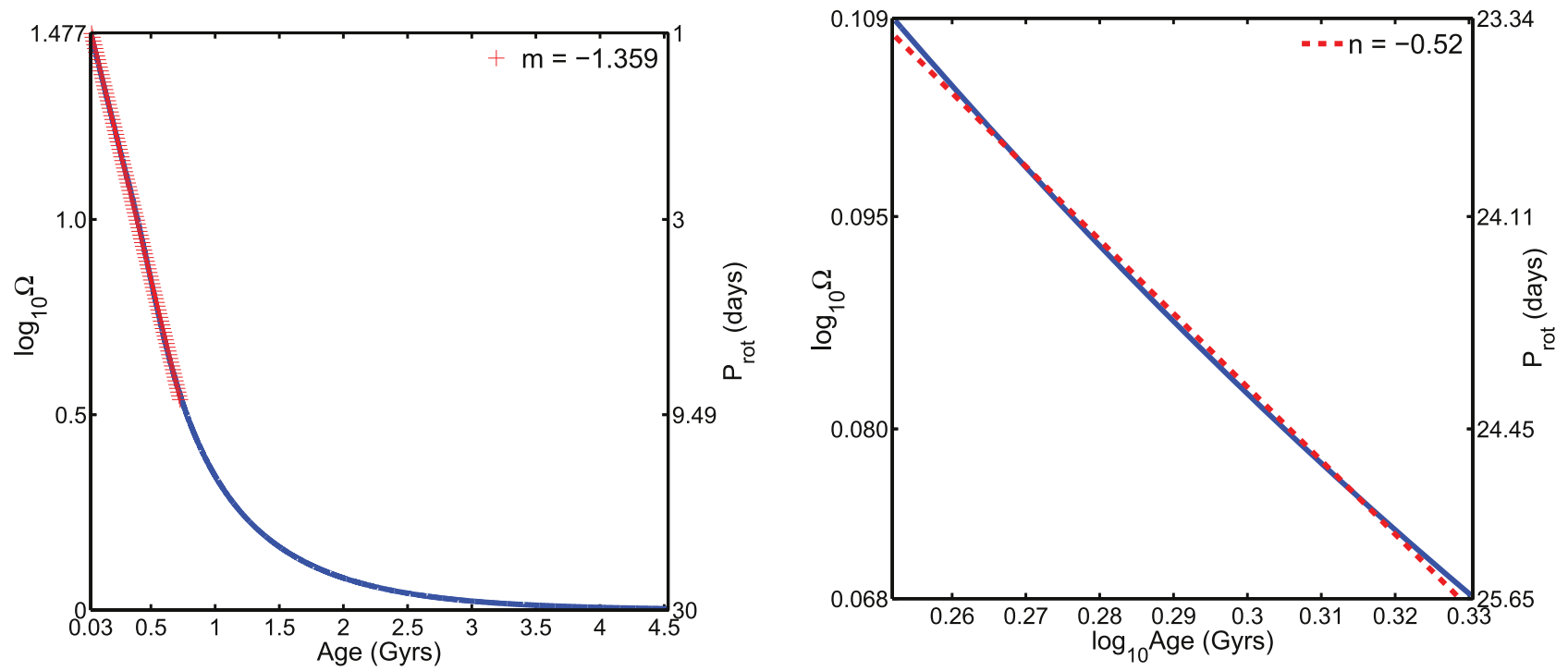

Figure 2. Left panel shows exponential spindown with $\Omega \propto \exp ^{-1.35 t}$ for ages $\in[0.03,0.7325]$ Gyr depicted in red, while blue represents the trend in semilogarithmic scale for age $\in[0.03,4.53]$ Gyr. The right panel shows the power-law spindown, $\Omega \propto t^{n}$, with scaling exponent -0.52 for solar-type stars. A gradual decrease in $|n|$ suggests a drop in the efficiency of angular momentum loss, which seems to align with the suggestion for the reduction in the efficiency of magnetic braking from recent observations from the Kepler space telescope (e.g., Garraffo et al. 2016).

the largely completed spindown process translates to the present-day age of the Sun of 4.5 Gyr.

To model the spindown process, we take the initial value of $\Omega$ to be 30 , corresponding to thirty times the present-day solar rotation, which is $\Omega=1$ in our non-dimensionalization. An initial value of $\Omega=30$ is intended to model the rotation rate of young stars at an age of around $\sim 10^{7}$ years. In contrast to $\Omega$, which can only decrease monotonically according to Equation (6), the initial conditions of the other four variables are not important, as they can increase as well as decrease, and turn out to settle into statistically stationary states on comparatively rapid timescales; that is, transients depending on the initial conditions of these quantities quickly vanish, and the subsequent evolution depends only on the initial value chosen for $\Omega$. Finally, note that because $\Omega$ is monotonically decreasing in time, we can effectively invert the relationship $\Omega(t)$ as $t(\Omega)$, and therefore consider all the other variables as functions of $\Omega$ rather than $t$.

\section{RESULTS}

\section{1. $\Omega$ Versus Age Relationship}

Figure 1 shows the relationship between $\Omega$ and $t$. A sharp decrease in $\Omega$ can be seen for earlier times, which slows down as age starts increasing. In the left panel of Figure 2 we fit this curve using an exponential law, that is, $\Omega \propto e^{m t}$. The best fit, for stars with rotation periods in the range $1 \leqslant P_{\text {rot }} \leqslant 3$, has $m=-1.35$, corresponding to an $e$-folding time of $0.74 \mathrm{Gyr}$. In the right panel of Figure 2 we fit this curve using power laws, that is, $\Omega \propto t^{n}$. For larger times we get power-law scalings 
Table 1

Power-law Exponent $n$ for Stars with Different Rotation Period in Days

\begin{tabular}{llc}
\hline \hline$n$ & $\Omega$ & $P_{\text {rot }}($ days $)$ \\
\hline-1.38 & $\Omega \in[3.5,1.99]$ & $8.57-15$ \\
-0.97 & $\Omega \in[1.99,1.50]$ & $15-20$ \\
-0.70 & $\Omega \in[1.50,1.28]$ & $20-23.34$ \\
-0.52 & $\Omega \in[1.28,1.17]$ & $23.34-25.65$ \\
\hline
\end{tabular}

which vary gradually for different rotation rates, that is, $n$ becomes smaller for smaller rotation as observed in MacGregor $\&$ Brenner (1991). For larger ages (slower rotation rates), the power-law exponent $n$ is found to be around -0.52 for stars with rotation periods in the range $23 \leqslant P_{\text {rot }}<25.65$. For different rotation rates we summarize the scalings in Table 1.

\section{2. $|B|$ Versus $\Omega$ Relationship}

Magnetic field strength $|B|$ is shown as a function of rotation rate in Figure 3 (left panel). The unit of $B$ is normalized by the strength of the magnetic field in the present-day Sun, which is roughly of order $10^{4} \mathrm{G}$ in the solar tachocline and $3 \mathrm{G}$ in the atmosphere. Figure 3 exhibits notably different behavior of $|B|$ in the two different rotation rate regimes. For slow rotation rates, we can clearly see the increasing behavior of $|B|$ with rotation rate which attains a maximum value at $\Omega \approx 5.8$. For $\Omega \in[1.17,5]$, the scaling of $|B|$ with respect to $\Omega$ is found to vary between 2.73 and 0.36 . We observe an average scaling of 1.47 for $\Omega \in[1.25,2]$ which is close to the observed scaling of $1.38 \pm 0.14$ (Vidotto et al. 2014). We note that $|A|$ also scales with $\Omega$ similarly to $|B|$. Interestingly, there is a decrease in $|B|$ which continues up to $\Omega \simeq 12.5$. For $\Omega \geqslant 12.5$, that is, for very high rotation rates, $|B|$ fluctuates on a very rapid timescale, but with a cycle-averaged value, depicted in red, that is essentially independent of $\Omega$. The rapid fluctuations in $|B|$ are due to the presence of two modes with different frequencies. The fluctuating behavior of $|B|$ with $\Omega$ can be seen in Figure 3 (right panel) for a small cut of $\Omega \in[23.30,23.31]$. Note how the system spends more time near the top as opposed to the bottom, which explains why the cycle-averaged value of $|B|$ (the red curve in the left panel) is higher than the simple average of the cycle maxima and minima (the highs and lows of the blue curves). (Observationally this would suggest that stars might be more likely to be observed close to a peak of magnetic activity rather than a trough.) Furthermore, we notice a gap between the two different rotation rate regimes in the region $\Omega \in[5.8,12.5]$.

\subsection{Power Spectra of B and the $\omega_{\mathrm{cyc}}$ Versus $\Omega$ Relationship}

To understand how the rapid cycles in $|B|$ gradually evolve as $\Omega$ spins down, we divided the entire time series into discrete chunks of $0.0106 \mathrm{Gyr}$, and performed a Fourier transform on each chunk separately. The precise length of the individual sections is not important, the only requirements being that it should be long compared with the fast cycle time, but short compared with the gradual spindown evolution time. Figure 4 shows Fourier spectra for eight such sections. It is notable that at earlier times shown in the first and second rows, there are two main peaks around $\omega \sim 10$ in the spectra, whereas at later times there is only one in the third and fourth rows, with the peaks furthermore shifting to lower frequencies. In particular, in the second and third rows, where time increases from 0.1460 to $0.2831 \mathrm{Gyr}$, we find the peaks shifting gradually toward lower frequency as time increases. This behavior continues until we reach a time of approximately $0.3253 \mathrm{Gyr}$, beyond which the multiple peaks of frequency are found to diminish. This behavior of frequency can be seen in panel 7 of Figure 4 for time $\approx[0.3148,0.3253]$ Gyr while for time $\approx[0.3569$, 0.3675 ] Gyr we find only a single peak of frequency (see Figure 4 panel 8). The behavior of the power spectra of $|B|$ clearly shows that the second peak of frequency vanishes as time increases, that is, as the rotation rate decreases. We note that in addition to the two main peaks at $\omega \sim 10$ or $\omega<10$ that we discussed above, one or two more peaks are also observed at higher frequency $\omega \sim 20$ in the first and second rows. These high-frequency modes have much weaker power than the main peaks and are simply their subharmonics. In the following, we do not discuss these modes and only focus on the behavior of the main peaks (e.g., the higher-frequency modes are not shown in Figure 5).

The gradual transitions in the spectra of $|B|$ are further illustrated in Figure 5, showing so-called short-time Fourier transforms. In this technique the signal is again divided into short chunks, but these now overlap, essentially forming a moving window, and hence giving an overview of the continuous evolution of frequencies and amplitudes. Using this method, the most pronounced frequency of $|B|$ is obtained in Figure 5 (left panel) where high to low intensity of frequency is illustrated via bright red to dark blue colors as shown in the color map. For early times $t<0.3253 \mathrm{Gyr}$, we observe two curves of frequency of maximum intensity $\omega_{\text {cyc }}$ (depicted in red) with age in Gyr. The lower curve has a larger amplitude of frequency than the upper curve. The existence of these two curves is the manifestation of complex time behavior of fast rotators and is reminiscent of the complexity of magnetic topology for active branch stars, discussed in recent papers (e.g., Matt et al. 2015). Both upper and lower curves show that the frequency of maximum intensity decreases with age rapidly until $t \sim 0.3253 \mathrm{Gyr}$ when the upper curve disappears while the lower curve exhibits a change in behavior. This single curve for $t>0.3253 \mathrm{Gyr}$ is interpreted as an inactive branch.

In order to investigate further, we examine the scaling of $\omega_{\text {cyc }}$ by showing the behavior of frequency of maximum intensity $\omega_{\text {cyc }}$ against rotation rate $\Omega$ in the right panel in Figure 5 . Again, we notice that for fast rotation rate we have two curves of frequency for maximum intensity, whereas for slow rotation rate we have only a single curve. We use a power-law relationship, that is, $\omega_{\text {cyc }} \propto \Omega^{p}$ with a power-law index $p$ to obtain the scaling. For the upper curve, we find the value of $p \sim 0.83$ for stars with rotation rate $12.8 \leqslant \Omega \leqslant 30$. On the other hand, the scaling exponent $p$ of the lower curve varies with rotation rate, as shown in Table 2. Interestingly, for fast rotators with $\Omega>12$, an average value is $p \sim 0.9$, which is close to the observational value for active branch stars (Saar \& Brandenburg 2001); for slow rotators, solar-like stars with rotation rate in the range $[1.17,3.5]$ have $p \sim 1.16$, in good agreement with observed scaling exponent for solar-type stars lying on the inactive branch (Saar \& Brandenburg 2001).

\subsection{Total Shear Versus $\Omega$ Relationship}

In our dimensionless units, the total shear is given by $1+w_{0}$. Figure 6 shows how this total shear changes with rotation rate $\Omega$. As $\Omega$ increases from $\Omega=1$, the total shear is seen to decrease by $90 \%$ from 1 to 0.1 with increasing $\Omega$. This 

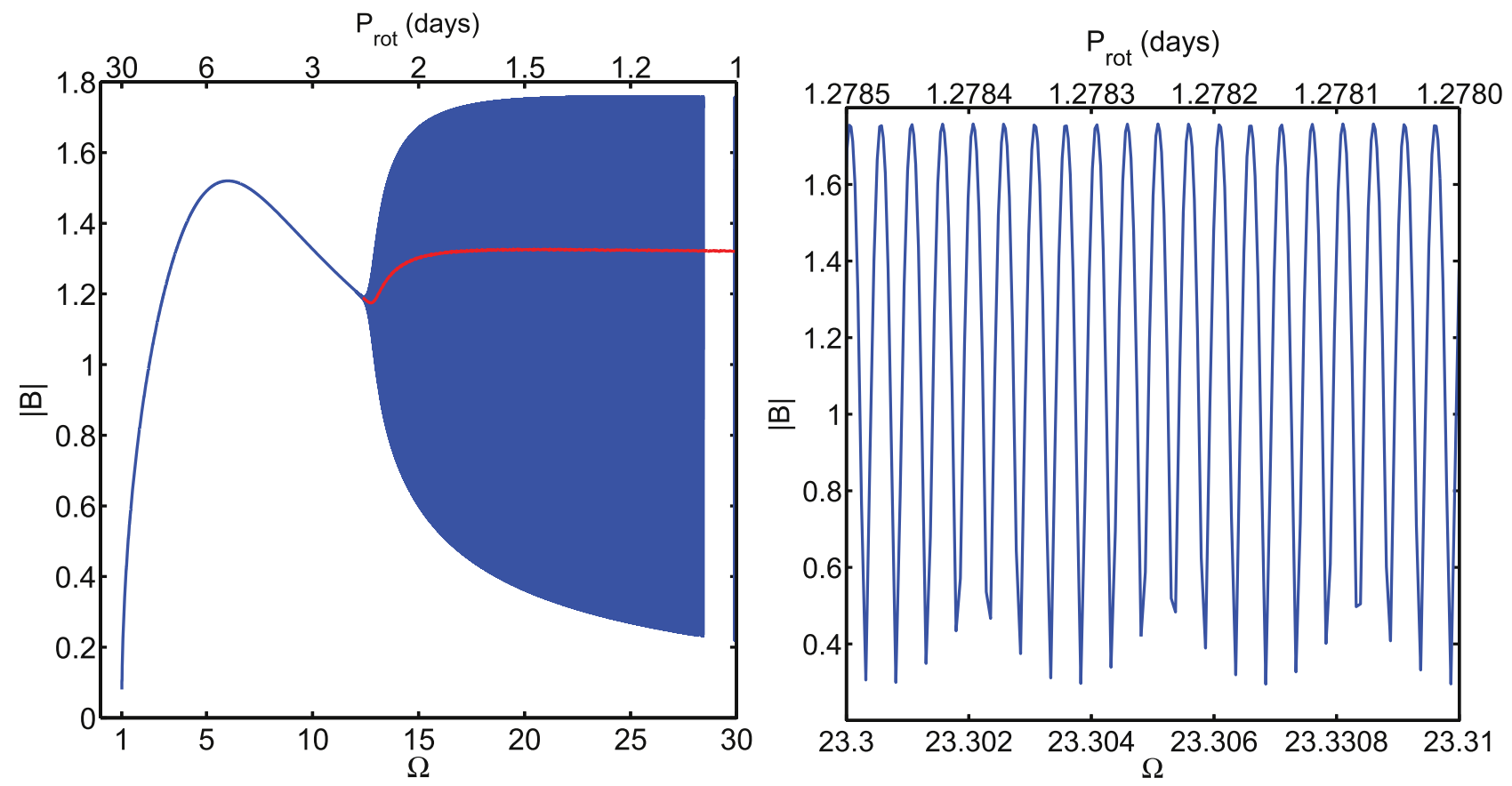

Figure 3. Magnetic field strength $|B|$ as a function of $\Omega$ (left panel) with the cycle-average of $|B|$ depicted in red. We note that the value of $|B| \approx 0.1$ at $\Omega=1$ corresponds to about $3 \mathrm{G}$ at the star's surface, whereas the rotation period $\left(P_{\mathrm{rot}}\right)$ in days is depicted on the top of the plot. Also, the fluctuating behavior of $|B|$ for the high rotation rate regime is shown for $\Omega \in[23.30,23.31]$ (right panel).

reduction in total shear results from the effect of magnetic back-reaction on the shear. The saturation of the total shear for high rotation indicates that the dynamo efficiency is not saturated beyond a certain rotation rate. After taking the minimum value around $\Omega=12.5$, the total shear increases with $\Omega$ in a small interval $\Omega \in[12.5,17]$ and then remains almost constant for high rotation rate $\Omega \geqslant 17$. It is important to note that the apparently broad band of the total shear for $\Omega \geqslant 12.5$ in Figure 5 is due to the two different modes with different frequencies existing in this interval. The inset in Figure 6 shows the total shear for a small range of $\Omega \in[29.82,29.84]$ to highlight the fluctuation due to the two modes. Finally, $\Omega=12.5$, where the total shear takes its minimum value, is related to a very rapid transition in rotational evolution and to the $V-P$ gap discussed later.

\section{5. $|B|$ Versus Age Relationship}

In Figure 7 magnetic field strength is shown as a function of age. The magnetic field strength $|B|$ is observed to maintain almost the same mean value fluctuating with finite amplitude for very young fast rotating stars of age up to $325 \mathrm{Myr}$. This fluctuation is due to the presence of the two different modes, as discussed below. The magnetic activity is seen to increase with age in the range $\in[325,502] \mathrm{Myr}$ as $|B| \sim t^{s}$ with a power-law exponent $s=0.53$ after which the magnetic activity remains almost constant in the age interval $\in[508,551]$ Myr. Beyond this value the magnetic activity decreases very rapidly with increasing age. We find that the power-law exponent $s$ varies with different values for stars with different ages, and these variations are provided in Table 3. Finally, we note that our results suggest that the fraction of poloidal versus toroidal flux fluctuates for fast rotators, consistent with their complex magnetic topology (e.g., Matt et al. 2015), while it takes a constant value for slow rotators. The mean value of this ratio does not change significantly over time.

\subsection{Timescale for Spindown}

In order to quantify the timescale for spindown, we compute the characteristic spindown time $\tau=|\Omega / \dot{\Omega}|$ using Equation (6) as we evolve the system, and then show the suitable averaged value in Figure 8 using linear and $\log$ scales. The inset in Figure 8 (left panel) shows the zoomed-in view of $\tau$ for very fast rotating stars. Here, red depicts the mean value ${ }^{3}$ of timescale over time. Clearly, we observe a spindown timescale of $15.96 \mathrm{Myr}$ for very young, rapidly rotating stars of ages from $30 \mathrm{Myr}$, which decreases very slowly up to age $315 \mathrm{Myr}$. Beyond this, the spindown timescale is observed to decrease rapidly with increasing age for a short interval [315, 493.9] Myr. This decline in spindown time reaches a minimum of approximately $122 \mathrm{Myr}$ for age $493.9 \mathrm{Myr}$. After this, the spindown timescale starts increasing with the age of the stars. Specifically, the spindown timescale increases linearly for solar-type stars with ages approximately $4.5 \mathrm{Gyr}$. The shortest spindown timescale is obtained in the region $[315,632] \operatorname{Myr}(\Omega \in[5.8,12.5])$ noted previously, and interestingly corresponds to the $V-P$ gap, the transition region between fast and slow rotators. That is, this is the region where the star suddenly jumps from the active to inactive branch, staying in this intermediate region for a short time only due to the fast spindown. To summarize, our results show that the spindown time for fast rotating stars in that region is shorter than the spindown time for slow rotating stars while the spindown timescale for stars in the transition region is even shorter than the spindown timescale for fast rotating stars.

\footnotetext{
3 This mean value is obtained over the $\frac{1}{420}$ fraction of the interval.
} 

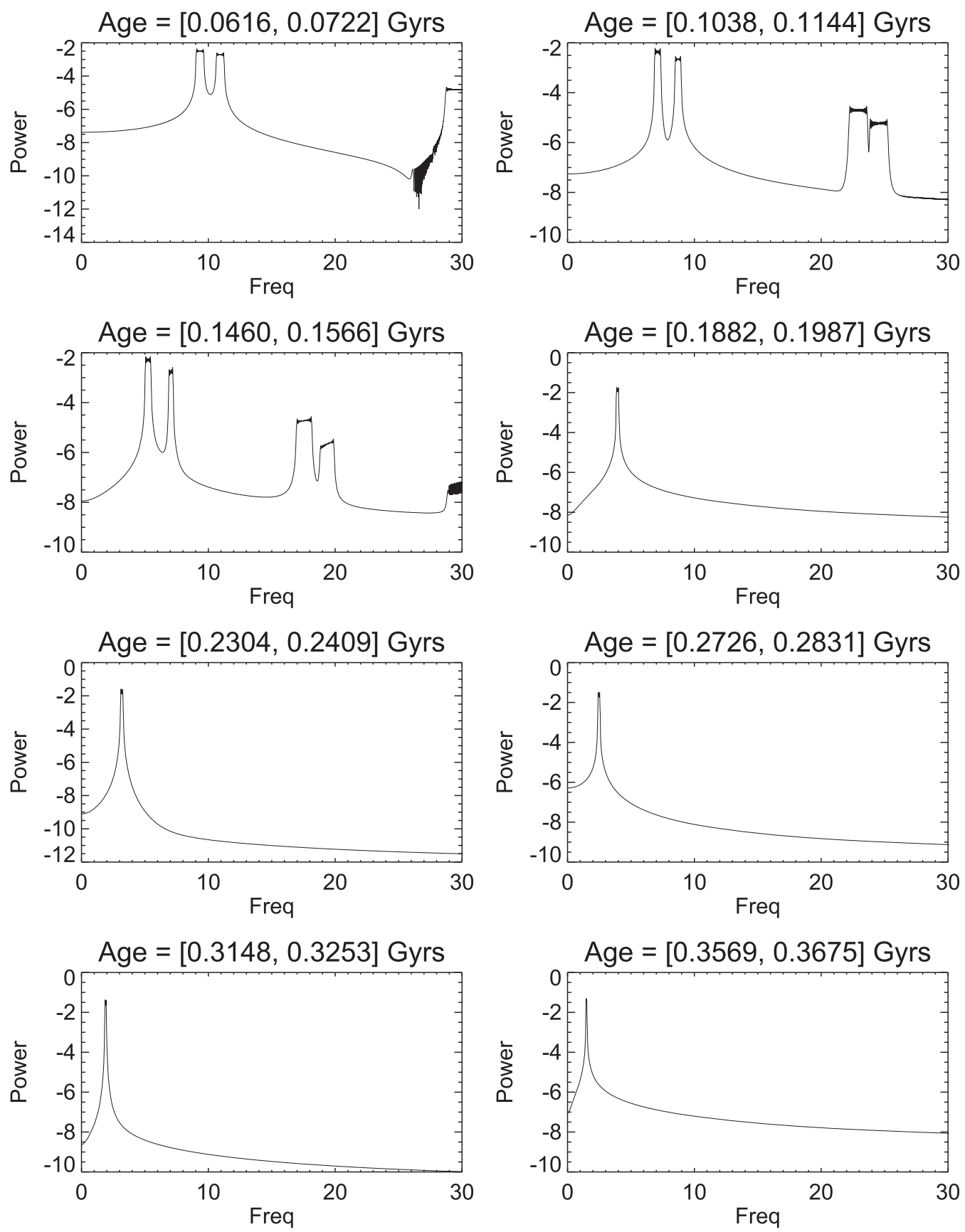

Figure 4. Power spectra of $|B|$ for eight distinct intervals in time, indicated by the numbers at the top of each panel.

These results are in good agreement with observations for spindown timescale (Barnes 2003).

\subsection{Summary of Results}

Our dynamical model of spindown coupled to the evolution of magnetic fields successfully reproduced: (i) the basic $\Omega$ versus age relationship, (ii) the relationships of $|B|, \omega_{\text {cyc }}$ and total shear $1+w_{0}$ versus $\Omega$, and (iii) the magnetic activity and spindown timescales with age. All three results are consistent with observations for the spindown process for fast and slow rotating stars, and provide a natural explanation for the $V-P$ gap associated with the abrupt transition of stars from the active (A) to the inactive $(I)$ branch, which is an important unresolved issue.

\section{CONCLUSIONS}

The evolution of magnetic fields and rotation rate is a selfregulated process through the direct interaction between largescale shear flow and magnetic field, and the indirect interaction of various (nonlinear) feedback mechanisms through smallscale fields. In particular, the generation of magnetic field and spindown are closely inter-linked processes since the generation of the former depends on the rotation of stars and thus spindown, while the latter process crucially depends upon the magnetic field (e.g., generation, destruction) and differential rotation. In this paper, we have proposed a dynamical model of spindown to understand the self-regulation of magnetic fields and rotation over the spindown timescale, which for the first time evolves the magnetic field and rotation rate 

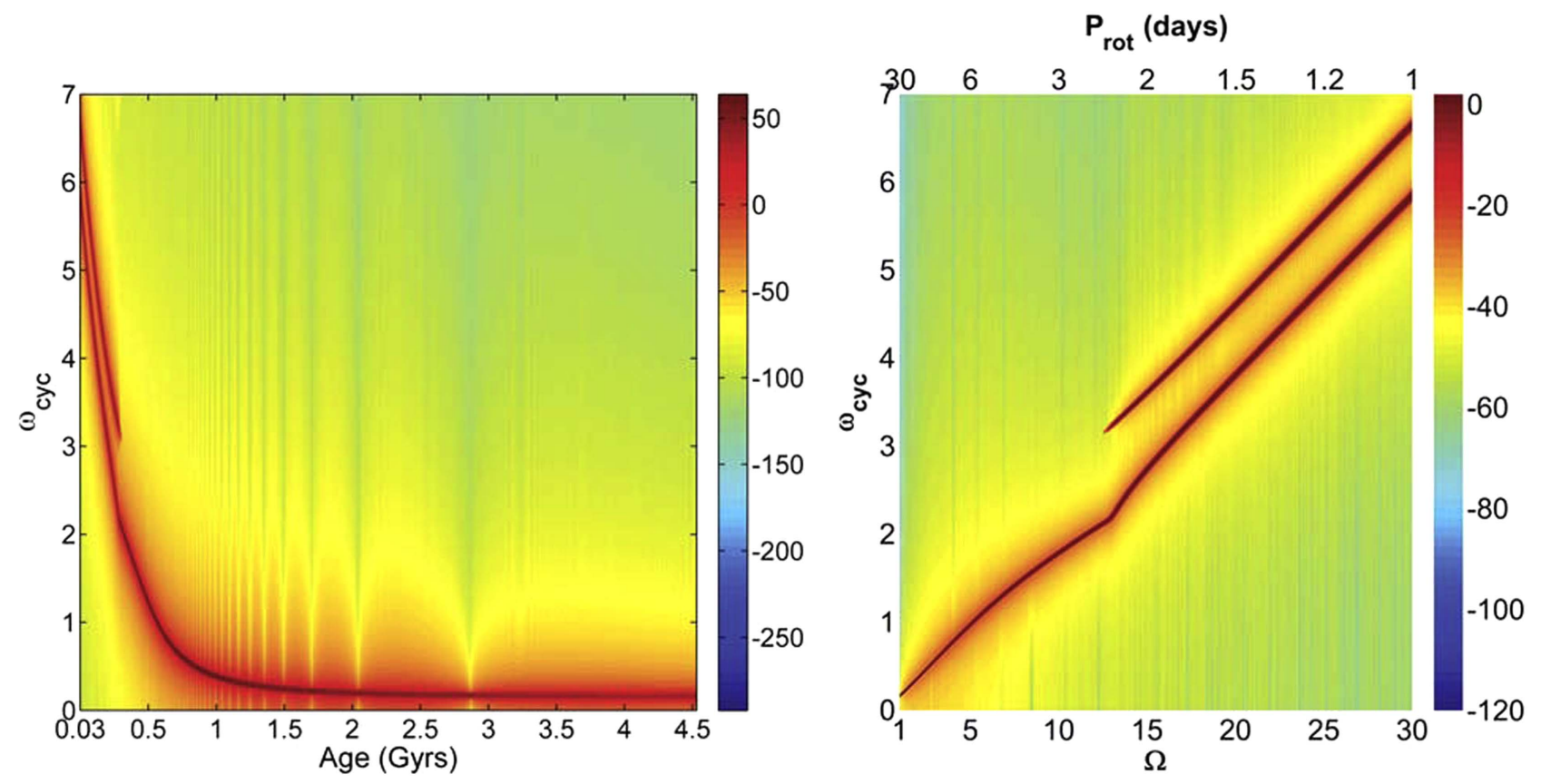

Figure 5. Behavior of frequency of maximum intensity $\omega_{\mathrm{cyc}}$, as a function of age in Gyr and rotation rate can be seen in left panel and right panel, respectively. Here, bright red to dark blue colors represent high to low intensity of frequency.

Table 2

Power-law Exponent $p$ for the Lower Curve in $\omega_{\text {cyc }} \propto \Omega^{p}$ at Different Rotation Periods

\begin{tabular}{llc}
\hline \hline$p$ & $\Omega$ & $P_{\text {rot }}($ days $)$ \\
\hline 1.16 & $\Omega \in[1.17,3.5]$ & $25.65-8.7$ \\
0.98 & $\Omega \in[3.5,6]$ & $8.7-5$ \\
0.80 & $\Omega \in[6,13]$ & $5-2.30$ \\
1.06 & $\Omega \in[16,30]$ & $1.88-1$ \\
\hline
\end{tabular}

\section{$P_{\text {rot }}$ (days)}

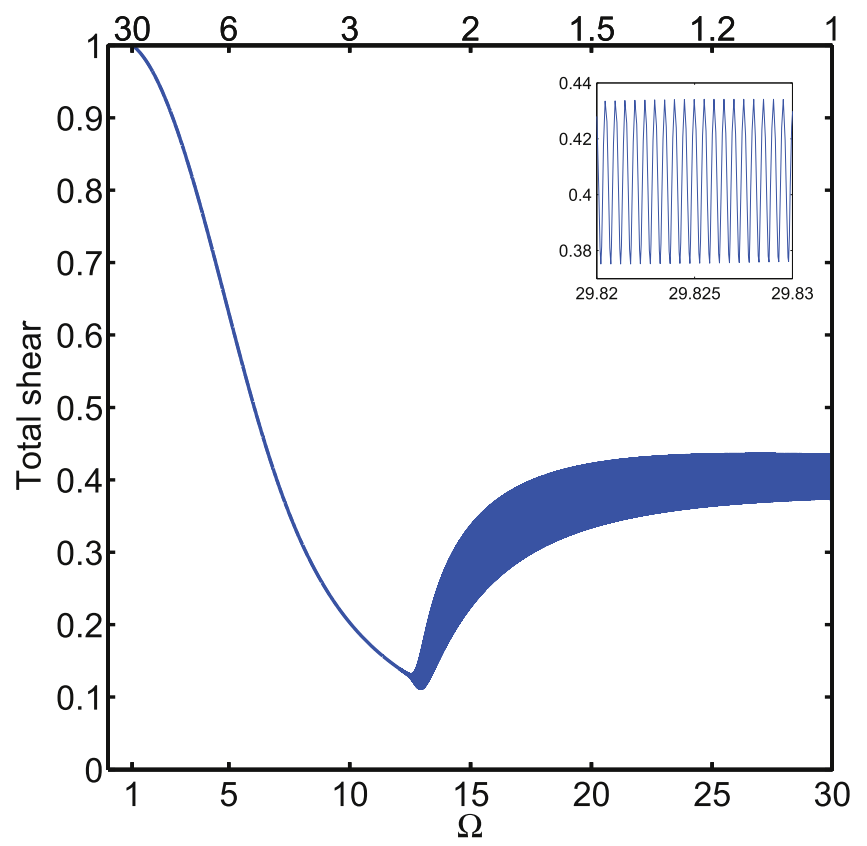

Figure 6. Total shear $1+w_{0}$ as a function of rotation rate $\Omega$.

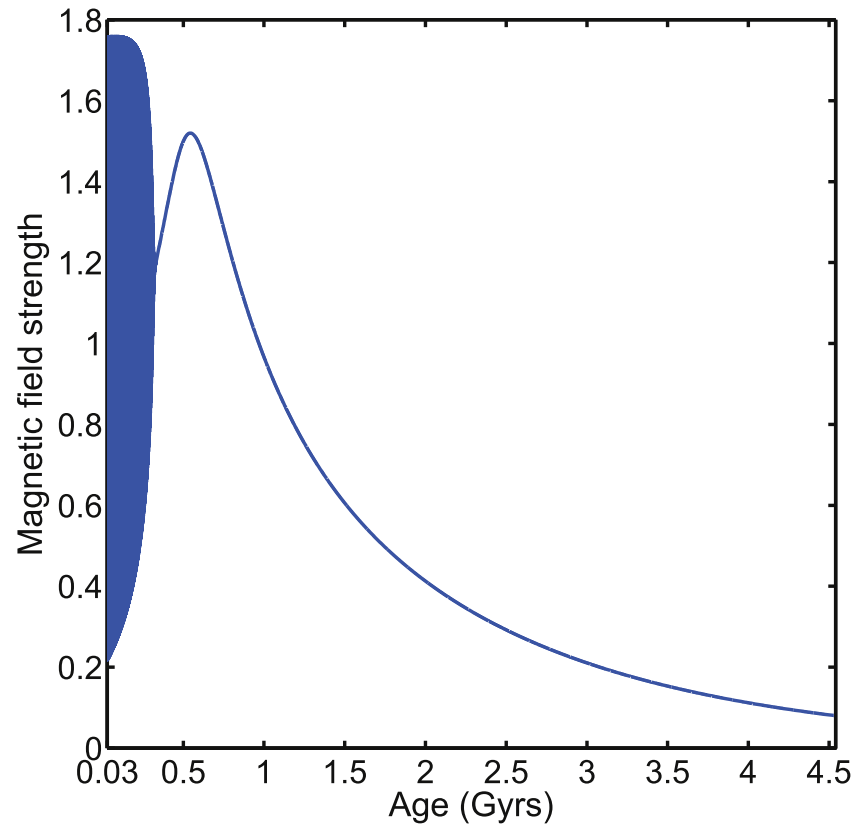

Figure 7. Magnetic field strength $|B|$ as a function of age.

Table 3

Power-law Exponent $s$ for Magnetic Activity with Age $t$ of Stars $\in[1.066,4.5] \mathrm{Gyr}$

\begin{tabular}{lc}
\hline \hline$s$ & Age (Gyr) \\
\hline-0.61 & $t \in[0.5929,0.7336]$ \\
-0.97 & $t \in[0.7336,1.085]$ \\
-1.13 & $t \in[1.085,1,437]$ \\
-1.25 & $t \in[1.437,1.788]$ \\
-1.40 & $t \in[1.788,2.139]$ \\
-1.64 & $t \in[2.139,2.843]$ \\
-2.00 & $t \in[2.843,3.54]$ \\
-2.50 & $t \in[3.54,4.5]$ \\
\hline
\end{tabular}



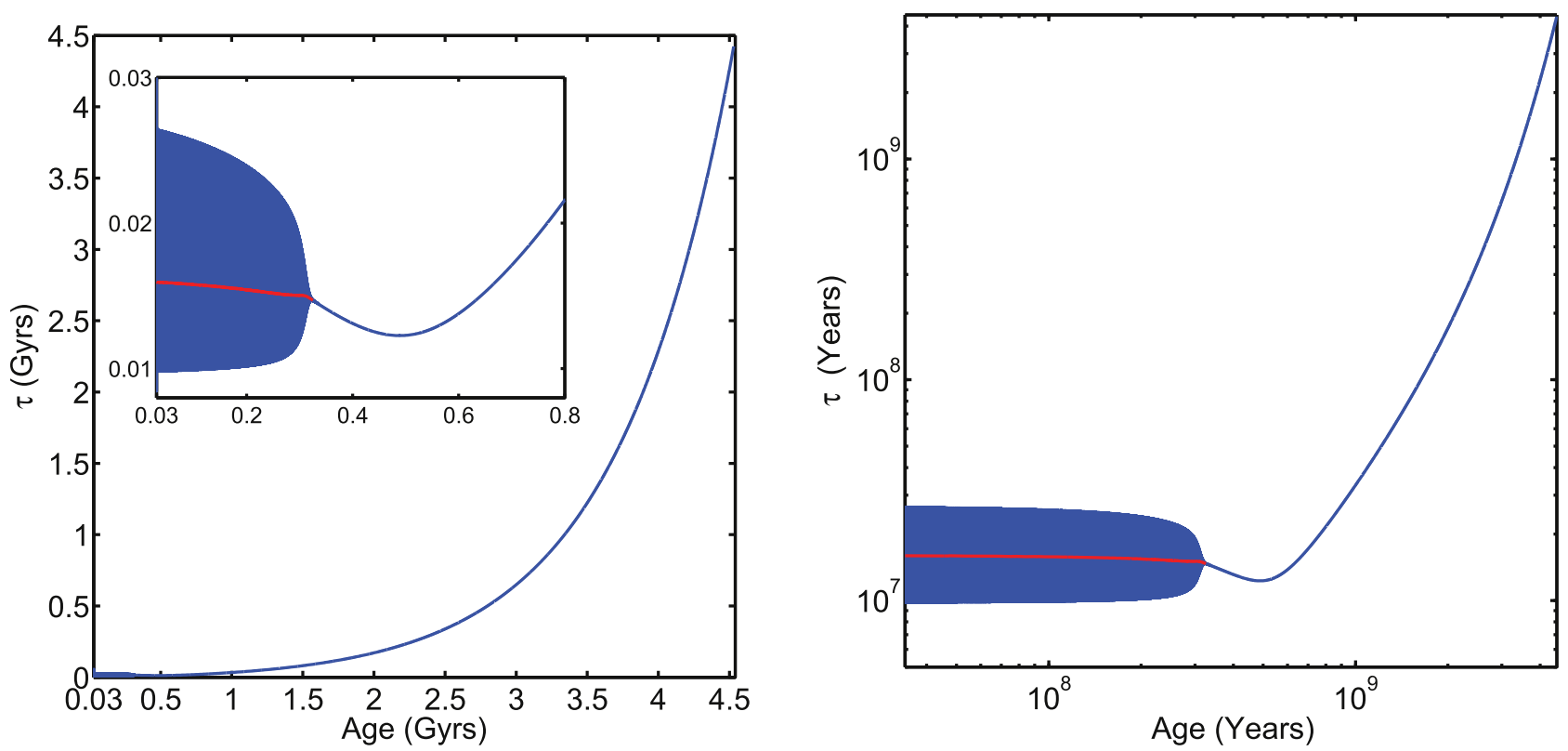

Figure 8. Left panel shows the spindown timescale $\tau$ as a function of age in Gyr in linear scale while right panel shows $\tau$ as a function of age in years in log-scale. The oscillations in $\tau$ are caused by the fluctuations in $\dot{\Omega}$ previously seen in Figure 1 (right panel).

simultaneously taking into account various mutual interactions. Despite being a simple parameterized model, it successfully reproduces the observations for spindown of stars which would otherwise be impossible in a more complete model (e.g., 3D MHD). In particular, we have found exponential spindown, saturation of magnetic field strength, and power-law dependence of frequency of magnetic fields of the active and inactive branches for rapidly rotating stars. For slow rotators, we obtained a power-law spindown, linear scaling of magnetic field strength, and a power-law relationship of $\omega_{\text {cyc }}$ on $\Omega$ with power-law scaling for the inactive branch. The transition from fast to slow rotating stars is quantitatively shown to occur very rapidly, thereby providing a natural explanation for the $V-P$ gap. In future, an interesting extension of our model would include coupling to the evolution of mass-loss (e.g., Garraffo et al. 2015) and detailed modeling of slow rotating stars (van Saders et al. 2016) and the transient Sun (Metcalfe et al. 2016).

We thank Dr. M. Miesch for valuable discussions.

\section{REFERENCES}

Barnes, S. A. 2003, ApJ, 586, 464

Barnes, S. A. 2010, ApJ, 722, 22

Barnes, S. A., \& Kim, Y-C. 2010, ApJ, 721, 675

Belcher, J. W., \& MacGregor, K. B. 1976, ApJ, 210, 498

Böhm-Vitense, E. 2007, ApJ, 657, 486

Brandenburg, A., Saar, S. H., \& Turpin, C. R. 1998, ApJ, 498, 51

Brown, T. M. 2014, ApJ, 789, 101

Charbonneau, P., \& Saar, S. H. 2001, in ASP Conf. Ser. 248, Magnetic Fields across the H-R digram, ed. G. Mathys, S. K. Solanki, \& D. T. Wickamasinghe (San Francisco, CA: ASP), 189

Cohen, O., Drake, J. J., Kashyap, V. L., \& Gombosi, T. I. 2009, ApJ, 699, 1501

Cranmer, S. R., \& Saar, S. H. 2011, ApJ, 741, 54

Denissenkov, P. A., Pinsonneault, M., Terndrup, D. M., \& Newsham, G. 2010, ApJ, 716, 1269

Dikpati, M., \& Charbonneau, P. 1999, ApJ, 518, 508

Donati, J.-F., \& Cameron, A. C. 1997, MNRAS, 291, 1
Durney, B. R., Mihalas, D., \& Robinson, R. D 1981, PASP, 93, 537

Epstein, C. R., \& Pinsonneault, M. H. 2014, ApJ, 780, 159

Gallet, F., \& Bouvier, J. 2013, A\&A, 556, A36

Garraffo, C., Drake, J. J., \& Cohen, O. 2015, ApJ, 813, 40

Garraffo, C., Drake, J. J., \& Cohen, O. 2016, A\&A, in press (arXiv:1607. 06096v1)

Irwin, J., \& Bouvier, J. 2009, in IAU Symp. 258, The Ages of Stars, ed. E. E. Mamajek, D. R. Soderblom, \& R. F. G. Wyse (Cambridge: Cambridge Univ. Press), 363

Johnstone, C. P., Güdel, M., Lüftinger, T., Toth, G., \& Brott, I. 2015, A\&A, 577, A27

Kawaler, S. D. 1988, ApJ, 333, 236

Keppens, R., MacGregor, K. B., \& Charbonneau, P. 1995, A\&A, 294, 469

Krishnamurthi, A., Pinsonneault, M. H., Barnes, S., \& Sofia, S. 1997, ApJ, 480,303

Leprovost, N., \& Kim, E. 2010, ApJ, 719, 287

MacGregor, K. B., \& Brenner, M. 1991, ApJ, 376, 204

Mamajek, E. E., \& Hillenbrand, L. A. 2008, ApJ, 687, 1264

Matt, S. P., Brun, A. S., Baraffe, I., Bouvier, J., \& Chabrier, G. 2015, ApJL, 799, L23

Matt, S. P., MacGregor, K. B., Pinsonneault, M. H., \& Greene, T. P. 2012, ApJL, 754, L26

Mestel, L. 1968, MNRAS, 138, 359

Mestel, L., \& Spruit, H. C. 1987, MNRAS, 226, 57

Metcalfe, T. S., Egeland, R., \& van Saders, J. 2016, ApJL, 826, L2

Micela, G., Sciortino, S., Serio, S., et al. 1985, ApJ, 292, 172

Noyes, R. W., Weiss, N. O., \& Vaughan, A. H. 1984, ApJ, 287, 769

Pallavicini, R., Golub, L., Rosner, R., \& Vaiana, G. S. 1981, ApJ, 248, 279

Pizzolato, N., Maggio, A., Micela, G., Sciortino, S., \& Ventura, P. 2003, A\&A, 397, 147

Reiners, A., \& Mohanty, S. 2012, ApJ, 746, 43

Réville, V., Brun, A. S., Matt, S. P., Strugarek, A., \& Pinto, R. F. 2015, ApJ, 798,116

Saar, S. H. 2002, in ASP Conf. Ser. 223, The 11th Cool Stars: Stellar System and the Sun, ed. R. J. Garcia Lopez, R. Rebolo, \& M. R. Zapatero (San Francisco, CA: ASP), 292

Saar, S. H., \& Brandenburg, A. 1999, ApJ, 524, 295

Saar, S. H., \& Brandenburg, A. 2001, in ASP Conf. Ser. 248, Magnetic Fields across the Hertzsprung-Russell Diagram, ed. G. Mathys, S. K. Solanki, \& D. Wickramasinghe (San Francisco, CA: ASP), 231

Scholz, A. 2008, arXiv:0810.1190

Skumanich, A. 1972, ApJ, 171, 565

Sood, A., \& Kim, E. 2013, A\&A, 555, A22

Sood, A., \& Kim, E. 2014, A\&A, 563, A100 
Spada, F., Lanzafame, A. C., Lanza, A. F., Messina, S., \& Collier Cameron, A. 2011, MNRAS, 416, 447

Tassoul, J.-L. 2000, Stellar Rotation (Cambridge: Cambridge Univ. Press) van Saders, J. L., Ceillier, T., Metcalfe, T. S., et al. 2016, Natur, 529, 181

Vaughan, A. H., \& Preston, G. W. 1980, PASP, 92, 385
Vidotto, A. A., Gregory, S. G., Jardine, M., et al. 2014, MNRAS, 441, 2361

Weber, E. J., \& Davis, L., Jr. 1967, ApJ, 148, 217

Weiss, N. O., Cattaneo, F., \& Jones, C. A. 1984, GApFD, 30, 305

Wright, N. J., Drake, J. J., Mamajek, E. E., \& Henry, G. W. 2011, ApJ, 743, 48 